\title{
Presencia femenina en los espacios deportivos televisivos
}

\author{
Female presence in television sports
}

\author{
Dick Ronald Cáceres Navarro* \\ Luis Alberto Vásquez Ampudia \\ Escuela Profesional de Ciencias de la Comunicación, \\ Universidad de San Martín de Porres, Perú
}

\section{Resumen}

Una preocupante realidad se vive en el interior de la prensa deportiva debido a la poca importancia que se le da a la presencia de la mujer dentro de este género periodístico. El predominio masculino que rige en estos tipos de programas convierte a la mujer en un «objeto» de atracción para incrementar los niveles de audiencia; modalidad recurrente no solo en el Perú, sino en otros países del orbe, producto del estereotipo del concepto de la mujer.

El ámbito deportivo siempre ha sido un terreno dominado por los hombres y causa sorpresa que en el siglo actual, donde impera la tecnología y la informática y supuestamente la sociedad tiene otra mentalidad, se mantengan pensamientos machistas, que desmerecen el trabajo que vienen realizando las mujeres para desarrollarse a nivel personal y profesional en una sociedad que le es adversa y que no respeta sus derechos.

El objetivo de la presente investigación es demostrar que las mujeres tienen un bajo nivel de presencia en los segmentos deportivos de los noticieros matutinos de América Televisión (Primera Edición), Panamericana Televisión (Buenos Días Perú) y ATV (ATV Edición Matinal).

Palabras clave: medios de comunicación, periodismo, deportes, mujer.

Este es un artículo Open Access bajo la licencia Creative Commons Atribución-NoComercial-Compartirlgual 4.0

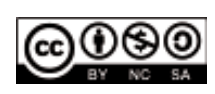




\begin{abstract}
A worrying situation occurs inside the sports press, due to the little importance given to women in this journalistic genre. The predominance of men in these types of programs makes women an "object" of attraction for increasing audience engagement: a recurrent modality adopted not only in Peru but in other countries around the world, as a result of female stereotyping.
\end{abstract}

Sports have always been a field dominated by men. It is surprising that, in the current century, where technology and computer science prevail, and society has supposedly changed its mentality, macho thoughts are still present, detracting the work being done by women to develop personally and professionally in a society that is adverse to them and does not respect their rights.

The aim of this research is to demonstrate that women have little presence in the sports segments of the news broadcasts of America Television (Primera Edición [First Edition]), Panamericana Televisión (Buenos Días, Perú [Good Morning, Peru]) and ATV (ATV Edición Matinal [ATV Morning Edition]).

Keywords: media, journalism, sports, woman.

\title{
Introducción
}

Después de 36 años el Perú volvió a participar en un mundial de fútbol, y la expectativa fue tan grande que diariamente se hablaba de ello, sobre todo en los programas deportivos de la televisión de señal abierta, en los que se desplegaron equipos humanos y técnicos para la cobertura de dicho evento.

Precisamente, dentro de estos equipos, destacó la abrumadora presencia de los hombres, y si bien participaron mujeres, las labores que desempeñaron fueron extradeportivas o consideradas de menor rango.

Para graficar lo expuesto se presenta tres casos:

1. En Utero.pe, se analiza la participación femenina en el más reciente programa deportivo que la cadena Fox Sports produjo en el Perú: 
A esta corte de caballeros futboleros se les suma la presencia de una sola mujer. Sentada en un rincón frente a un computador, la Miss Perú 2018, Romina Lozano, se encarga de ser «la voz de las redes» en el programa. «Rominita», como la llama el moderador del debate, Matías Brivio, debe esperar el permiso de este para poder hablar. Por supuesto, Romina no está ahí para opinar, como todos los demás, su rol es leer los tuits de lo que piensan los televidentes, quienes son casi todos hombres. (Grados, 2018)

2. El periodista chileno Loreto Santibáñez recoge las declaraciones de la bloguera peruana María José Osorio donde comenta las preguntas que se le hacen en un programa deportivo a una corresponsal en Rusia. También recoge las declaraciones de Giuseppina Lobos, periodista de El Gráfico de Chile, quien sostiene:

Esta es una realidad en la mayoría de los programas deportivos. Las mujeres presentes son más bien un adorno para leer redes sociales. Según mi punto de vista es un error de ambos, de los productores y directores por todavía mantener esa visión machista con respecto a nuestra labor en el deporte, y de nosotras mismas por aceptar esas condiciones y no ofrecer un «pack» más completo. Eso se puede negociar: «Ok, yo leo las redes sociales, pero también tú me das la oportunidad de hablar y dar mi opinión cuanto se esté discutiendo un tema». También va en la proactividad que nosotras tengamos. (como se citó en Santibañez, 2017)

3. Victoria Scarioni Avellaneda en su trabajo titulado Periodismo de taquito. La mujer en el periodismo deportivo, contextualiza la actual situación de la mujer periodista dentro de los programas deportivos televisivos:

Generalmente se presenta a la mujer desde un lugar que busca satisfacer las necesidades o expectativas de los espectadores, ya que es usual que aparezca una mujer joven y con buena apariencia física, de pie y tratando temas pocos importantes o transmitiendo lo que los espectadores opinan en las redes sociales. (Scarioni, 2018) 
Por consiguiente, a pesar de transitar ya en el siglo XXI, y con sociedades (supuestamente) avanzadas, sigue existiendo muy arraigadamente el machismo y la discriminación hacia la mujer en todos los ámbitos, pero sobre todo, en el campo del periodismo deportivo, que fue y es históricamente territorio de hombres.

Los medios de comunicación contribuyen a la perpetuación de las formas jerárquicas de las diferencias de género, por medio de los intereses que predominan en el capitalismo y el patriarcado. Estos intereses, basados en la lógica de la máxima ganancia y el hombre económico, han defendido la dominación del cuerpo femenino mientras les ha producido beneficios. Estos medios juegan cotidianamente en sus mensajes con discursos sexistas, segregacionistas y discriminatorios.

Por lo tanto, esta investigación tiene como objetivo principal demostrar que las mujeres tienen un bajo nivel de presencia en los segmentos deportivos de los noticieros matutinos de América Televisión (Primera Edición), Panamericana Televisión (Buenos Días Perú) y ATV (ATV Edición Matinal).

El desempeño de la mujer en los programas deportivos de la televisión no solo se circunscribe al Perú sino también a otros países, la tendencia va siempre en la misma dirección: no otorgarle a la mujer el manejo y desempeño en labores que los hombres realizan.

\section{Periodismo deportivo}

El periodismo deportivo ha vivido en las últimas décadas un notabilísimo desarrollo en sus estructuras, canales y modos de producir contenidos, cobrando un creciente protagonismo que llega a afianzarse en el momento actual como la tipología informativa de mayor alcance social en una buena parte de los países tanto europeos como americanos. El florecimiento de esta tipología informativa no solo es numérico; marca tendencias, creando un estilo propio, fundamentado en el color, la originalidad y la espectacularidad de sus propuestas; aportando un nuevo modelo de presentación de las informaciones mediante un lenguaje visualmente atractivo que resulta común y accesible para todas las mentalidades. 
El florecimiento de este tipo de periodismo especializado se fundamenta en gran medida en el hecho de que los profesionales de estos medios «informan de un género específico comprensible a todas las mentalidades a través de un lenguaje universal que todos entienden, producto del espíritu y la filosofía del deporte, como fenómeno cultural más seguido y practicado desde comienzos del siglo pasado y que va en aumento en el siglo que hemos iniciado». Este auge ha propiciado que en nuestros días el periodismo deportivo sea una parcela de estudio de importancia creciente y que, cada vez más, adquiera un mayor reconocimiento dentro del mundo mediático (donde los reporteros deportivos ya no son vistos como outsiders) y de los ámbitos cultural y académico, a diferencia de antaño. (Alcoba, 2005, p. 10)

El periodismo deportivo tal como lo conocemos nace con el auge del periodismo especializado, cuya principal característica es que los profesionales de la información tratan de llevar las incidencias de las distintas disciplinas deportivas a la mayor cantidad de público que es conocedor de la filosofía deportiva.

Es inevitable que la academia tome al periodismo deportivo como objeto de investigación, habida cuenta que el culto por algunos deportes como el futbol convierte también a los periodistas de esta especialidad en influyentes líderes de opinión.

El periodista deportivo debe ser consecuente con el código ético para que refleje su imparcialidad. Debido al gran alcance de difusión de sus comentarios, está obligado a responder por la veracidad de su información para no poner su imagen en duda. Una inconsistencia, que detectan los aficionados, es la diferencia de criterios de algunos comentaristas sobre un encuentro deportivo.

$\mathrm{Al}$ respecto, Villena (2003) señala:

El periodismo deportivo hoy más que nunca es un show en el cual el público no solo aprecia la información como tal sino que busca la opinión de los periodistas especializados. Por este motivo 
creemos que la perspectiva femenina debe estar presente en la mayoría de estos espacios. En el periodismo deportivo actual, el deporte-juego ha dado paso al deporte-espectáculo, de manera que los intereses son más importantes que la opinión de los informadores y que llevan a comentarios que no tienen nada que ver con el espíritu y filosofía del deporte y llevarán una carga de peligrosidad de la cual él es el único responsable. (p. 130)

\section{Características de los formatos deportivos en la prensa televisiva nacional}

La prensa deportiva en nuestro país, así como en muchos a nivel global, se caracteriza por espectacularizar determinada información, que en el ámbito deportivo más purista puede resultar intrascendente, tal es así que muchas veces la presentación de resultados de las diversas disciplinas son desplazados por las anécdotas que ocurren antes y después de un partido, o información relacionada a la vida privada de los deportistas.

Haciendo hincapié en ello, Marín (2009) manifiesta:

Una de las características del periodismo deportivo es la sobredimensión de los acontecimientos, es decir, se le otorga excesiva importancia a determinados acontecimientos deportivos, de tal forma que se hace una noticia de un asunto sin tanta trascendencia. Esta sobredimensión de las anécdotas deportivas, casi siempre las relacionadas al fútbol, conlleva al desplazamiento de otros mensajes deportivos. El resultado es que se da más importancia a informaciones sobre las actividades más fútiles del día a día de las estrellas del balompié que a informaciones políticas o culturales, en perjuicio de la información más puramente deportiva. (p. 168)

Con la aparición de Internet la prensa deportiva no ha sido ajena a la multimedialidad de sus contenidos, por esta razón las grandes empresas informativas desarrollan un contenido deportivo tanto para radio, televisión, web y redes sociales. 
En la actualidad, la libertad de expresión ha quedado, en cierto modo, secuestrada por las empresas periodísticas, al convertirse en monopolios informativos que imponen su criterio a los medios que las forman, los cuales deben responder a las exigencias del patrón: Las empresas periodísticas potentes son multimedias que marcan las directrices a sus periódicos, emisoras de radio y cadenas de televisión, aunque en ocasiones se dan casos esperpénticos como que uno de los medios se prohíba escribir sobre un determinado deporte, el boxeo, salvo para denigrarlo, y en los otros se autorice. (Alcoba, 2005, p. 156)

\section{Presencia de la mujer en la prensa deportiva nacional}

Hay que destacar que en los últimos años la mujer tiene mayor presencia en el periodismo deportivo de nuestro país, sobre todo en roles relacionados con la producción; sin embargo, no es notoria la presencia femenina en áreas como la conducción, narración o reporterismo. En virtud de lo cual Palumbo sostiene:

Creo que hay más hombres que trabajan en el periodismo deportivo porque ellos son voraces consumidores de información deportiva, sobre todo de fútbol, que es el deporte que más espacio ocupa en los medios. Creo que una mujer informada en este rubro puede tentar una plaza sin problemas. De hecho, ya hay algunas que están desarrollándose en este ámbito destacadamente. Hay periodistas, productoras y comentaristas mujeres. Hay que tener en cuenta que el periodismo deportivo (todo tipo de periodismo, en realidad) no involucra únicamente a quienes aparecen en pantalla, sino que hay todo un equipo detrás que trabaja con la información. Hay editores de contenido, editores de video, productores de campo, productores de transmisiones, directores, reporteros, en fin. Y muchos de ellos son mujeres. (2015, como se citó en Peschiera, 2016, pp. 188-189)

Admite el avance de la presencia femenina en diversos espacios deportivos, sin embargo, se desprende de su opinión que en el campo de la conducción 
de estos formatos televisivos aún es predominante la presencia masculina sobre todo en el ámbito futbolístico.

En esta línea, Román refiere:

Hace treinta o cuarenta años no podíamos imaginar que existieran tantas mujeres inmersas en lo que se refiere al periodismo deportivo ... Tengo la suerte de haber nacido en una época donde se está aceptando más el papel de la mujer; ya no podemos hablar de una o de dos chicas en lo que se refiere al periodismo deportivo, ahora el abanico es mucho más amplio, podemos encontrar mujeres en los periódicos, en la televisión, en la radio y ya también comentando un poco de lo que es fútbol, el polideportivo ... Ha sido muy complicado que la mujer ingrese en estos ámbitos pero creo que se está logrando sobre todo con la preparación. (2015, como se citó en Peschiera, 2016, p. 193)

\section{El machismo en la prensa deportiva}

A lo largo de la historia, la supremacía del hombre sobre la mujer se extiende en todos los ámbitos de la sociedad, lo que ha desencadenado que el machismo sea una conducta generalizada y en muchos casos aceptada.

El machismo se puede definir como un conjunto de creencias, actitudes y conductas que descansan sobre dos ideas básicas: por un lado, la polarización de los sexos, es decir, una contraposición de lo masculino y lo femenino según la cual no solo son diferentes sino mutuamente excluyentes; por otro la superioridad de lo masculino en las áreas consideradas importantes por los hombres. De aquí que el machismo involucre una serie de definiciones acerca de lo que significa ser hombre y ser mujer, así como toda una forma de vida basada en ello. (Castañeda, 2019)

Las mujeres han vivido o viven, en determinados casos, a la sombra de la hegemonía masculina. Si bien es cierto que en los últimos años sus derechos se han reivindicado y su presencia dentro de actividades laborales propias 
de los hombres ha sido cuestionada, urge la necesidad de implementar cambios en los estereotipos y en la idiosincrasia de las personas.

En este sentido, para Caudillo y Cerna (2007):

Los seres humanos vivimos en una sociedad ideada por hombres en la cual, las mujeres son simples apéndices del varón y sus deseos, fantasías y necesidades. El mundo de unos y otras está separado y no hay formas de aproximación, la cual se da sólo a través de la irritabilidad y la agresión. (p. 88)

Como se ha mencionado anteriormente, el público que gusta de los espacios deportivos televisivos no solo busca información, también está ávido de conocer el punto de vista de los periodistas dedicados al deporte. Llama la atención que la cara visible de los segmentos deportivos de los noticieros televisivos se encuentre a cargo de varones. Covarrubias (2009) destaca:

En gran parte de los programas deportivos, si bien es cierto se considera la presencia del sexo femenino, no hay mujeres entre los conductores y si es que las hay son solo el objeto sexual del programa o tal vez, solo hace entrevista de temas poco trascendentes.

Considerar a la mujer como objeto sexual o trasladarla a realizar entrevista de poca importancia confirman la imagen estereotipada que tienen de esta, en la que la consideran poco preparada para asumir esos temas y su gran aporte es solo la imagen que proyectará.

Con relación a esta problemática existen algunos teóricos que atribuyen este desbalance (entre conductoras y conductores deportivos televisivos) a un machismo predominante. Por ejemplo, según López (2011):

Si lo masculino está valorado y lo femenino infravalorado, los modelos sociales de identidad masculina y femenina que los medios construyen refuerzan valores tradicionales sobre la superioridad y dominación masculinas y la inferioridad y subordinación femeninas. (p. 76) 
Profundizando en el análisis, esta situación pone en evidencia la cultura patriarcal de nuestras sociedades, por lo que investigadores como Fraga (2006) aconsejan aplicar la equidad de género en el ámbito deportivo televisivo. Sostiene lo siguiente:

Otro aspecto importante de los medios es que son suministradores de la cultura dominante patriarcal y en esa medida conforman y difunden modelos de comportamiento sexista. $Y$ en tanto que son creadores de ideología es fundamental y necesario que se aplique una perspectiva de género sobre ellos, a través de la cual se detecten los sesgos y problemas que afectan a las mujeres en tanto que somos actoras y consumidoras de ellos. (p. 5)

Por otra parte, un problema por atender en la sociedad actual es el de la discriminación cuyas consecuencias afectan el entorno personal y social del ser humano.

Es una conducta, culturalmente fundada, sistemática y socialmente extendida, de desprecio contra una persona o grupo de personas sobre la base de un prejuicio negativo o un estigma relacionado con una desventaja inmerecida, y que tiene por efecto (intencional o no) dañar sus derechos y libertades fundamentales. (Rodríguez, 2005, p. 23)

La posición de la mujer en la sociedad no es ajena a la discriminación, todo lo contrario, además la violencia física y psicológica que enfrenta se ha acentuado en los últimos años. Se refleja hostilidad por parte de la sociedad dominada por un machismo que minimiza la labor de la mujer e impide su realización.

La vida de la mujer en la familia reclama el análisis de singulares formas de discriminación, desde la invisibilidad del trabajo doméstico que no se considera trabajo productivo, hasta la violación matrimonial y la servidumbre al marido y los hijos. No ha sido sino en estas últimas décadas que ha sido posible quebrar la sacralidad adjudicada a la familia para describir los distintos 
órdenes de violencia producidos en ella contra la mujer. (Giberti, 1995, p. 189)

La exclusión femenina en los segmentos deportivos televisivos es solo una extensión de la aparente desigualdad que ocurre en las distintas disciplinas deportivas, terreno en el cual las mujeres, aunque destaquen por sus logros, siguen siendo relegadas en lo que respecta a salario y reconocimiento social.

Es evidente la diferencia con el género masculino. Según Pedraza (2012):

Las dificultades se duplican cuando a las barreras para acceder al espacio público (perteneciente a los hombres) que supone el periodismo, se le añade el problema de acceder a un espacio simbólico (también perteneciente a los hombres) que supone el deporte, es decir, el periodismo deportivo se constituye como un espacio de doble exclusión. Al igual que en las canchas y pistas, las periodistas deportivas han conquistado lugares a lo largo de las décadas, y aunque siguen en franca desventaja, el número de mujeres que trabaja en esta área no ha dejado de aumentar. (p. 57)

\section{Método}

Se realizó una encuesta o sondeo de opinión a 100 personas, mayores de 18 años, hombres y mujeres, con residencia en Lima, televidentes de los segmentos deportivos de los noticieros matutinos de América Televisión (Primera Edición), Panamericana Televisión (Buenos Días Perú) y ATV (ATV Edición Matinal). Utilizando medios digitales y redes sociales se les formuló la siguiente pregunta:

¿Cree usted que los espacios deportivos de los noticieros matutinos de América Televisión (Primera Edición), Panamericana Televisión (Buenos Días Perú) y ATV (ATV Edición Matinal) tienen suficiente presencia femenina en la conducción? 


\section{Resultados y discusión}

En términos porcentuales, la muestra registra los siguientes resultados:

Frente a la pregunta icree usted que los espacios deportivos de los noticieros matutinos de América Televisión (Primera Edición), Panamericana Televisión (Buenos Días Perú) y ATV (ATV Edición Matinal) tienen suficiente presencia femenina en la conducción?, responden $95 \%$ no y $5 \%$ sí.

Al ser consultados sobre si consideran que debe otorgárseles más espacio a las mujeres dentro del periodismo televisivo deportivo, $60 \%$ señala que sí mientras que $40 \%$ opina lo contrario.

Para evaluar la preponderancia del machismo en el ámbito periodístico deportivo nacional se preguntó: iestá usted de acuerdo en que predomina el machismo en los espacios deportivos de los noticieros matutinos de América Televisión (Primera Edición), Panamericana Televisión (Buenos Días Perú) y ATV (ATV Edición Matinal)?, 85\% responden sí y 15\% responden no.

No obstante, al consultar si prefieren ver a mujeres en la conducción de programas periodísticos, $70 \%$ señaló que no, mientras $30 \%$ que sí.

Frente a la consulta sobre la preparación que tienen las mujeres para la conducción de los segmentos deportivos: ¿cree usted que las mujeres están preparadas para conducir programas deportivos en la televisión de señal abierta?, 35\% responden sí y $65 \%$ responden no.

Los integrantes de la muestra de la investigación, de sexo masculino, manifestaron que hace mucho tiempo el periodismo deportivo dejó de ser exclusividad de los hombres y que las mujeres van ganando espacios en los programas deportivos de la televisión como reporteras y comentaristas. Muchas de ellas destacan por su belleza y cautivan a los televidentes con el trabajo que realizan. Agregan que son pocas las mujeres que estudian o investigan acerca de un determinado tema, no se documentan bien y creen que es suficiente solo con leer el diario del día; además, señalan que los medios de comunicación, intentando mostrar una mejor imagen, buscan chicas con cara bonita y buen físico, prevaleciendo esto en muchos casos, antes que una buena formación profesional. 
Finalmente, las integrantes de la muestra de la investigación (sexo femenino) respondieron que la mentalidad machista propia del periodismo deportivo está cambiando, pero que aún falta mucho camino por recorrer; $\mathrm{y}$ que mantienen la esperanza en que llegue el día que exista igualdad de oportunidades para ambos sexos, donde la mujer también pueda demostrar sus capacidades profesionales.

\section{Conclusiones}

- El público es consciente de que la presencia femenina en los segmentos deportivos matutinos aún es insuficiente y considera que las profesionales de la información especializadas en deportes deben tener más espacios.

- La mayoría de personas del grupo a las que se les solicitó su opinión atribuyen esta situación, de las mujeres en los espacios deportivos, al machismo.

- Pese a esta realidad, que desfavorece a las mujeres en los espacios deportivos matutinos, los consultados no muestran demasiado interés en ver mujeres en la conducción de estos formatos audiovisuales.

- Aunque las profesionales dedicadas al periodismo deportivo han ganado espacios en la televisión, aún el público identifica a los hombres en la conducción de estas secuencias televisivas. 


\section{Referencias}

Alcoba, A. (2005). Periodismo deportivo. Síntesis.

Castañeda, M. (2019). El machismo invisible. Penguin Random House.

Caudillo, C. y Cerna, M. (2007). Sexualidad y vida humana. Universidad Iberoamericana.

Covarrubias, R. (2009). El ejercicio profesional de la mujer dentro del periodismo deportivo, desde la visión de jefes, productores, periodistas, comentaristas y aficionados de Guadalajara, Jalisco. Razón y Palabra, 14(69), 1-16.

Fraga, C. (2007). Las mujeres y los medios de comunicación. Una relación controvertida. Revista Comunicación e Cidadania, (1), 45-52.

Giberti, E. (1995). La discriminación de la mujer en América Latina. Ciclos, 5(9),185-191.

Grados, L. (2018, 2 de mayo). Para Fox Sports Perú las mujeres solo sirven de adorno y estas son las pruebas. Utero.pe. http://utero.pe/2018/05/02/para-fox-sports-peru-las-mujeres-solo-sirven-de-adorno-y-estas-son-laspruebas/

López, P. (2011). Deporte y mujeres en los medios de comunicación. Sugerencias y recomendaciones. Consejo Superior de Deportes del Gobierno de España.

Marín, J. (2009). Imagen, comunicación y deporte. Editorial Visión Libros.

Peschiera, L. (2016). El ejercicio profesional de la mujer dentro del periodismo deportivo. Correspondencias \& Análisis, (6), 185-196. https://doi.org/10.24265/cian.2016.n6.10

Pedraza, I. (2012). Mujeres en el periodismo deportivo: Reflexiones para comprender la transgresión desde la práctica discursiva de las reporteras de deportes. Derecho a Comunicar, (4), 47-69.

Rodríguez, J. (2005). Definición y concepto de la no discriminación. El Cotidiano, (134), 23-29.

Santibañez, L. (2017). Mujeres en el periodismo deportivo: ¿son usadas como adorno? El Mostrador. https:/l www.elmostrador.cl/braga/2017/06/28/mujeres-en-el-periodismo-deportivo-son-usadas-como-adorno/

Scarioni, V. (2018). Periodismo de taquito. La mujer en el periodismo deportivo. Economía feminista. http:/l economiafeminita.com/periodismo-de-taquito-la-mujer-en-el-periodismo-deportivo/

Villena, S. (2003). El fútbol y las identidades: Prólogo a los estudios latinoamericanos. En P. Alabarces, Futbologías: Fútbol, identidad y violencia en América Latina (pp. 130-133). CLACSO. 